\title{
Ozonetherapy In The Treatment Of Nocturnal Tinnitus
}

\author{
Juan Carlos Rivera Acosta ${ }^{1 *}$ \\ María Mónica Alejandra Cárdenas Moreno', \\ Juan Diego Céspedes Hurtado², \\ Mauricio Miguel Meza Delgado ${ }^{3}$ \\ Jessica Milena Santos Sierra ${ }^{4}$ \\ Dina Rafaela González Hernández ${ }^{4}$
}

\begin{abstract}
This systematic review was carried out in order to highlight the implementation of ozone therapy as a treatment for nocturnal tinnitus. It is important to note that ozone treatment has resolved nocturnal tinnitus with $100 \%$ efficacy in previous studies, producing in turn a symptomatic improvement in the patient. From these results, a high degree of efficacy can be inferred in overcoming nocturnal tinnitus treated with ozone therapy in patients with a severe index of perception of tinnitus.
\end{abstract}

Keywords: Ozone therapy, Tinnitus, Nocturnal tinnitus 


\section{INTRODUCTION}

Ozone therapy is known as a type of alternative medicine involved in the treatment of many chronic diseases (cancer, HIV, multiple sclerosis, etc.). Its main objective is the recovery of oxygen saturation in the body, through the insufflation of a mixture of oxygen and ozone to the body in various ways.

The fact that ozone at a controlled dose can exert antioxidant effects represents a therapeutic resource of great value in the treatment of multiple diseases that manifest themselves with a weakening of the endogenous antioxidant system. As a response to the introduction of ozone into tissues and organs, the compensatory increase occurs above all in the activity of antioxidant enzymes such as: superoxide dismutase, catalase and glutathione peroxidase, which are widely represented in the heart muscle, the liver, erythrocytes and other tissues.

Tinnitus is a symptom, not a disease, of a malfunction in the processing of information, at the level of the central nervous system with individual emotional reactions of greater or lesser quantity, already described since ancient times and that currently affects a part important part of the population.

The presence of tinnitus affects the individual at various levels. In the first place, it supposes an interference in the intelligibility of the auditory message, in addition, tinnitus is generally accompanied by hearing loss, so the corresponding audiological study will have to be carried out. It produces changes in lifestyle, as well as the presence of avoidance behaviors in situations or environments that involve a greater perception of the problem. Tinnitus can be accompanied by other symptoms and sometimes the cause that produces it requires prolonged drug treatments that can lead to dependence.

In addition, the lack of effective treatment to control the problem and inadequate (negative) medical advice can lead to frustration, irritability, feelings of helplessness, fear, anxiety and sometimes depression in patients. It is thought that the greater or lesser severity of the effects of tinnitus will depend on the psychoacoustic characteristics (intensity, frequency, level of masking).

A bibliographic search was carried out that spanned from 2017 to 2021 in the databases pubmed, Elsevier, scielo, Update, medline, national and international libraries. We use the following descriptors: Ozone therapy, tinnitus, nocturnal tinnitus. The data obtained oscillate between 16 and 60 records after the use of the different keywords. The search for articles was carried out in Spanish and English, it was limited by year of publication and studies between 2017 and 2021 were used. The main exclusion criteria were articles that had more than 5 years of publication.

\section{RESULTS}

Although until now there is no consensus as to how to face the cure for nocturnal tinnitus, there are several treatments available that can give different degrees of relief, and among them are those proposed by Dr. Carlos Herraiz from the Unit of tinnitus of the Otorhinolaryngology Service of the Alcorcón Foundation University Hospital in Madrid: 1) Counseling aimed at reducing stress. 2) Stress Management and Relaxation. 3) Diet therapy. 4) Emotional support groups. 5) Auditory Habituation. 6) Medications: there is no single medication that is effective in all patients with tinnitus. Antidepressants, anxiolytics, anticonvulsants, steroids (systemic or intratympanic) are used, which can improve the symptom and have been shown to be successful in a very small percentage of cases $^{1}$

In one of the published studies it was found that when medicinal ozone is introduced into the body, through rectal insufflation, it is captured by the inferior, middle and superior hemorrhoidal venous plexuses found in the rectum, and thus comes into contact. with venous blood. It dissolves in the water present in the venous plasma, where it reacts in seconds producing, by acting as a highly oxidizing agent, in a controlled oxidative stress that at therapeutic doses is capable of rebalancing the altered state in the body ${ }^{2}$.

The studies with the most evidence on the implementation of ozone therapy in the treatment of nocturnal tinnitus coincide in rectal insufflation. In a study carried out in an 88-year-old male patient, in which the diagnostic criteria used were based on the evolution of nocturnal tinnitus through the clinical semiology presented by the patient at the time of consultation, subjective perceptions and reports of this and the Tinnitus Severity Index Questionnaire. The treatment carried out lasted twenty rectal ozone therapy sessions whose frequency corresponded to that indicated in the current protocols and in which the doses $(1 \mathrm{mg}$ to $2.5 \mathrm{mg}$ of Ozone, progressively) used also followed the same guidelines established in them, but adapting to the results observed during the study ${ }^{3}$.

Regarding the field of otorhinolaryngology, ideas are raised about ozone treatment, in addition to conventional therapy, for patients with conditions of possible vascular etiology such as tinnitus, peripheral vertiginous syndrome, sudden hearing loss and others of infectious origin such as chronic otitis media. recurrent suppuration, rebellious to conventional therapy, which using the transrectal route has demonstrated the effectiveness of ozone treatment (91\%) without signs of intoxication or intolerance; Rapid remission of dizziness was observed in almost all patients from the first sessions and clear remission or improvement of tinnitus and hearing loss ${ }^{4}$.

\section{DISCUSSION}

Tinnitus When using Ozone therapy as a treatment for nocturnal tinnitus, two reactions or stages are observed, these reactions are distinguished in an initial phase or stage and a late one. In the initial reaction phase, a significant part of the administered ozone is consumed, due to the action of the antioxidants present in the plasma (which also decrease temporarily), forming a quantity of 
reactive oxygen species (ROS). In the late phase, the products formed from the initial phase, such as hydrogen peroxide, diffuse into the cells, activating the metabolic pathway of erythrocytes, leukocytes, and platelets. In red blood cells it activates the glycolysis pathway, increasing the concentration of the enzyme 2,3-diphosphoglycerate, increasing the final production of energy, and causing a transfer of oxygen to the tissues that translates into a beneficial effect, since it acts by modulating nitric oxide levels and thus regulating vasodilation ${ }^{5}$.

In the patient, this oxygenating effect is manifested from their perception of greater vitality and energy, as well as the increasing possibilities of movement and concentration. On the other hand, their statements regarding the possibility of achieving restorative sleep, the loss of their lethargy, and the recovery of agility to carry out cognitive activities, accounted for this property of ozone. As a metabolic regulator, preclinical and clinical observations have made it possible to assess the regulatory action of ozone on metabolic indicators that were initially in figures outside the normal ranges, such as glycemia; Cholesterolemia, creatinine; hemoglobin; blood pressure; liver enzymes; calcium; among others. This happens as part of the rebalancing or adjustment of the redox system. At the beginning of treatment, the perception of the intensity of tinnitus by the patient usually corresponds to the presence of severe tinnitus. Subsequently, the intensity decreases progressively and at the end of the treatment, the increasing decrease in tinnitus is complete, allowing a complete improvement physically and psychologically, even showing an improvement in the patient's mood.

\section{CONCLUSION}

Ozone treatment has resolved nocturnal tinnitus with $100 \%$ efficacy in previously conducted studies, in turn producing symptomatic improvement in the patient. From these results, a high degree of efficacy can be inferred in overcoming nocturnal tinnitus treated with ozone therapy in patients with a severe index of perception of tinnitus. There are still many blank spaces to establish ozone therapy as an alternative in the treatment of tinnitus. But based on the evidence already exposed and published it should be considered.

\section{CONFLICT OF INTEREST}

The authors declare that they have no conflicts of interest.

\section{REFERENCES}

1. Migliora M. Ozone Therapy in the Treatment of Night Tinnitus. Ozone Ther Global J. 2015;5:5-20.

2. Bhathal B, Alonso T, Pérez C, Poch B. Epidemiologicalclinical study on tinnitus in the field of ambulatory medicine. Acta Otorrinolaringol Esp. 1999;49:609-613.

3. Peña A. Physiopathological bases of the treatment of sensorineural tinnitus: Role of the efferent auditory system. J Otorhinolaryngo and Head and Neck Surgery. 2008;68:4958.

4. Schwartz A, Martinez-Sanchez G, Re L. Guide for the medical use of ozone. Therapeutic fundamentals and indications. Aepromo. 2011: 315.

5. Herraiz C, Plaza G, Aparicio JM, Gallego I, Marcos S, Ruiz C. Transtympanic steroids for Ménière's disease. Otol Neurotol. 2010;31:162-167. 